\title{
Outcomes observed during a 1-year clinical and radiographic follow-up of patients treated for 1- or 2-level cervical degenerative disease using a biodegradable anterior cervical plate
}

\author{
Mengcun Chen, MD, Shuhua Yang, MD, Cao Yang, MD, PhD, Weihua Xu, MD, PhD, \\ Shunan Ye, MD, PhD, Jing Wang, MD, PhD, Yong Feng, MD, PhD, Wen Yang, MD, PhD, and \\ Xianzhe Liu, MD, PhD
}

Department of Orthopaedics, Union Hospital, Tongji Medical College, Huazhong University of Science and Technology, Wuhan, People's Republic of China

OBJECTIVE The purpose of this study was to present an initial surgical experience in the management of 1- or 2-level degenerative disc disease of the cervical spine using biodegradable anterior cervical plates (bACPs) in anterior cervical discectomy and fusion (ACDF). The authors also aimed to provide insight into this critical and controversial clinical issue by clarifying outcomes for patients receiving bACPs and by comparing their outcomes with those achieved using a traditional metallic anterior cervical plate (mACP) implant.

METHODS A retrospective review was conducted for 2 series of patients who had undergone ACDF using either bACP (31 patients, 38 segments) or mACP (47 patients, 57 segments) instrumentation. The patients were followed up for a mean $13.5 \pm 0.9$ months (range 12-18 months) in the bACP group and $14.8 \pm 1.5$ months (range 14-22 months) in the mACP group. Clinical outcomes were determined according to scores on the visual analog scale (VAS), the modified Japanese Orthopaedic Association (mJOA) scoring system, and Odom's criteria. Radiological images were used to assess fusion rates, intervertebral height, Cobb's angle, and the width of prevertebral soft tissue.

RESULTS Both VAS and mJOA scores were significantly improved at each follow-up in both groups. Excellent or good results according to Odom's criteria were achieved in 93.5\% (29/31) of patients in the bACP group and 93.6\% (44/47) of patients in the mACP group. At 6 months postoperatively, the fusion rate was $94.7 \%(36 / 38)$ in the bACP group and $96.5 \%(55 / 57)$ in the mACP group, but subsidence of the intervertebral space at the surgical level was more evident in the bACP group. Angulation, as measured by Cobb's angle, demonstrated obvious healing in both groups, while better maintenance was observed in the mACP group. The local inflammatory reaction was uneventful during follow-up. Dysphonia and dysphagia were observed in both groups during the follow-up.

CONCLUSIONS The relatively comparable early clinical and radiographic outcomes and the overall acceptable complication rates for bACP and mACP use suggest that bACPs could be used as alternative instruments in ACDF. Mild graft resorption was noted without evidence of symptoms. However, the prospective efficacy of biodegradable instrumentation can only be elucidated with longer-term observation.

http://thejns.org/doi/abs/10.3171/2016.1.SPINE15807

KEY WORDS anterior cervical discectomy; biodegradable; lordosis; cervical spondylosis; spine surgery

$\mathrm{D}$ EGENERATION of the cervical spinal column is one of the most common disorders leading to neck or arm pain and neurological deficits. ${ }^{12}$ Anterior cervical discectomy and fusion (ACDF) is a classic procedure that has remarkable effects for this condition. Moreover, the utilization of an anterior cervical plate (ACP) is found to generate significantly more favorable outcomes. ${ }^{21} \mathrm{Howev}$ er, despite numerous advantages of the traditional titanium alloy implants, application of these materials is associated with several limitations. The rigidity and permanent pres-

ABBREVIATIONS ACDF = anterior cervical discectomy and fusion; $\mathrm{ACP}=$ anterior cervical plate; $\mathrm{bACP}=$ biodegradable $\mathrm{ACP} ; \mathrm{mACP}=\mathrm{metallic} \mathrm{ACP} ; \mathrm{mJOA}=\mathrm{modified}$ Japanese Orthopaedic Association; PVST = prevertebral soft tissue; VAS = visual analog scale.

SUBMITTED July 9, 2015. ACCEPTED January 8, 2016.

INCLUDE WHEN CITING Published online March 25, 2016; DOI: 10.3171/2016.1.SPINE15807. 
ence of the metallic implant may lead to short- and longterm complications. ${ }^{11,19}$ Also, the artifacts generated by the metallic alloy can obscure the interpretation of postoperative images. ${ }^{2}$

A biodegradable ACP (bACP) was introduced to overcome these limitations. The relatively less rigid biodegradable material more closely matches the physiological elastic modulus of the vertebra and results in greater load transfer to the interbody grafts to promote bony union. ${ }^{6}$ Better postoperative radiographic imaging evaluations can be conducted using a bACP because of its radiolucency. Additionally, the inherent biodegradability can extinguish the incidence of long-term migration or local erosion. Since the introduction of bACPs, many authors have investigated the feasibility of implantation in vitro and in vivo..$^{-4,8,9,13,15,16,18,20,23,24}$ Although most of these studies reported promising results, several more recent studies expressed concerns regarding inadequate stability, incompatible properties, and inferior clinical manifestations. ${ }^{3,4,15,16}$ The observational findings in the present study provide valuable insight into this critical and controversial clinical issue by clarifying outcomes for bACP use and by comparing these outcomes with those achieved for standard metallic ACP (mACP) implants, to evaluate the potential for bACPs to serve as an alternative to mACPs.

\section{Methods}

After obtaining approval from the local ethics committee, we conducted a retrospective review of a consecutive series of 31 patients (38 segments) who had undergone ACDF involving bACP instrumentation between October 2012 and March 2014 in the orthopedic department of Wuhan Union Hospital. A consecutive series of 47 patients (57 segments) who had undergone the same procedure with an mACP were included as a control group. The patients' demographic data, including age, sex, and some pathological conditions, are provided in Table 1.

The indications for ACDF surgery were as follows: 1) intolerable neck or brachial pain after a series of conservative remedies; 2) radiculopathic symptoms such as weakness in the hands or indicative Hoffman sign; and 3) gait disturbance or walking abnormality, with well-defined corresponding MRI-documented signs of degeneration (e.g., foramina stenosis and disk herniation). The exclusion criteria were as follows: 1) severe ossification of the posterior longitudinal ligament, 2) compression mainly behind the vertebral body or cervical canal stenosis, and 3) active infection, tuberculosis, or tumor.

\section{Implants Used}

The cage was made of polyetheretherketone (PEEK) with a central perforation inserted with mixed auto and allograft bone, allowing for subsequent bony ingrowths. The height of the cage varied from 5 to $8 \mathrm{~mm}$.

The titanium alloy implant was the ABC anterior cervical plating system (Aesculap Implant Systems LLC). The plate was constrained but could be bent to the patient's individual lordosis, and the screw for monocortical fixation was a titanium, self-tapping, conical screw ranging from 10 to $18 \mathrm{~mm}$ in length with an outer diameter of 4.0
TABLE 1. Basic and clinical patient characteristics

\begin{tabular}{lccc}
\hline \multicolumn{1}{c}{ Characteristic } & bACP Group & mACP Group & p Value \\
\hline No. of patients (segments) & $31(38)$ & $47(57)$ & - \\
\hline Sex (male/female) & $18 / 13$ & $19 / 28$ & 0.127 \\
\hline Mean age in yrs ( \pm SD) & $49.3 \pm 7.5$ & $53.3 \pm 6.4$ & $<0.05$ \\
\hline No. of smokers & 7 (all male) & 11 (all male) & 0.947 \\
\hline
\end{tabular}

$-=$ not applicable.

$\mathrm{mm}$. The single-level plates ranged from 20 to $32 \mathrm{~mm}$ in length, and the double-level plates ranged from 34 to 58 $\mathrm{mm}$ in length.

The biodegradable plate was the Inion S-1 biodegradable anterior cervical fusion system (Inion, Inc.), which comprised plates and screws made of degradable copolymers composed of $80 \%$ L-lactic acid and $20 \%$ DL-lactic acid (Fig. 1). The plate could be softened in an Inion Thermo+ water bath preheated to $70^{\circ} \mathrm{C}$ for approximately 1 minute and contoured to the desired shape as measured by a template.

Single-level plates are fabricated in 6 different sizes (lengths ranging from 21 to $31 \mathrm{~mm}$, with a width of $19 \mathrm{~mm}$ and a thickness of $2.0 \mathrm{~mm}$ ), whereas double-level plates range in length from 33 to $48 \mathrm{~mm}$ with the same width and thickness as the single-level plates. The diameters of the screws were 4.5 and $5.0 \mathrm{~mm}$ with lengths of 12,14 , and 16 $\mathrm{mm}$. Tantalum markers were set on the upper and lower end of the plates and in the screws.

\section{Surgical Technique}

The operation was performed with the patient supine with the neck slightly extended. A routine right-sided anterior Smith-Robinson approach was selected to reach the pathology. Complete decompression was achieved via the removal of the pathological protuberant fragments and part of the posterior longitudinal ligament. The cepha-
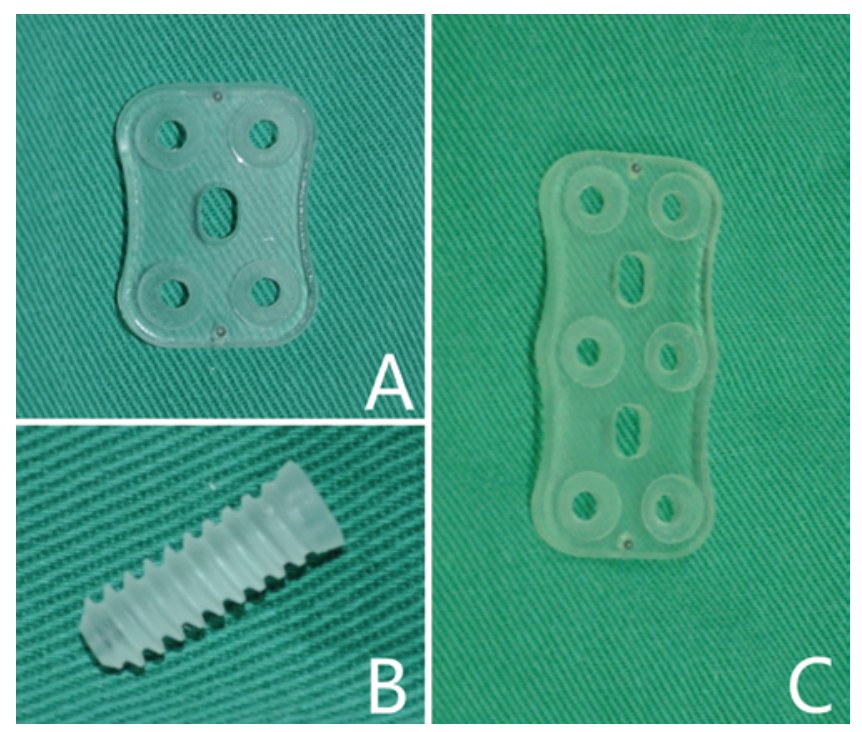

FIG. 1. The Inion S-1 biodegradable anterior cervical fusion system. Single-level plates (A), biodegradable screws (B), and double-level plates $(\mathbf{C})$ are shown. Figure is available in color online only. 
lad and caudal sides of the intervertebral area were prepared by removing the cartilaginous parts and thinning the cortical parts of the endplates to allow insertion of an intervertebral graft. After intervertebral grafting, a titanium plate (for the mACP group) was manually bent and placed in front of the vertebral body. In the bACP group, a template was first used to determine the appropriate size and shape. The corresponding plate was then submerged in hot water preheated to $70^{\circ} \mathrm{C}$ to be softened. The desired dimension was achieved by manually bending, and the customized plate hardened as it cooled and was secured to the vertebral bodies using $4.5-\mathrm{mm}$ biodegradable screws (length 12-16 mm).

Wound drainage systems were regularly used and removed 2 days after the operation. Postoperative collars were used for 4 weeks to ensure stability.

\section{Postoperative Follow-Up}

The patients were discharged 3-7 days postoperatively, depending on intraoperative bleeding and each patient's general condition. Patients were asked to return to our clinic for postoperative review at 1,3,6, and 12 months after surgery and then yearly thereafter. Clinical symptoms and signs and pre- and postoperative radiographs were recorded at each follow-up.

\section{Clinical Assessment}

Functional outcomes were assessed using the modified Japanese Orthopaedic Association (mJOA) scoring system and Odom's criteria.,17 A visual analog scale (VAS) scoring system was used to estimate neck and brachial pain separately. Dysphagia or foreign body sensation was recorded as an indication of the occurrence of sterile abscesses. Additional postoperative presentations such as pain, dyspnea, dysphonia, and other complications were noted as well.

\section{Radiographic Evaluation}

Cervical radiographs were taken at each follow-up, including routine anteroposterior and lateral views, whereas flexion-extension films were obtained at the 6-month and later postoperative follow-ups. The radiographs were used to evaluate bony fusion, pseudoarthrodesis formation, intervertebral height, segmental sagittal alignment, and prevertebral soft tissue (PVST) reaction at the operative level by using previously described methods (Fig. 2). 2,3,8,13,16,20 MRI of the cervical vertebrae was performed only when the patient complained of dysphagia.

\section{Statistical Analysis}

Data are expressed as the means \pm standard deviations

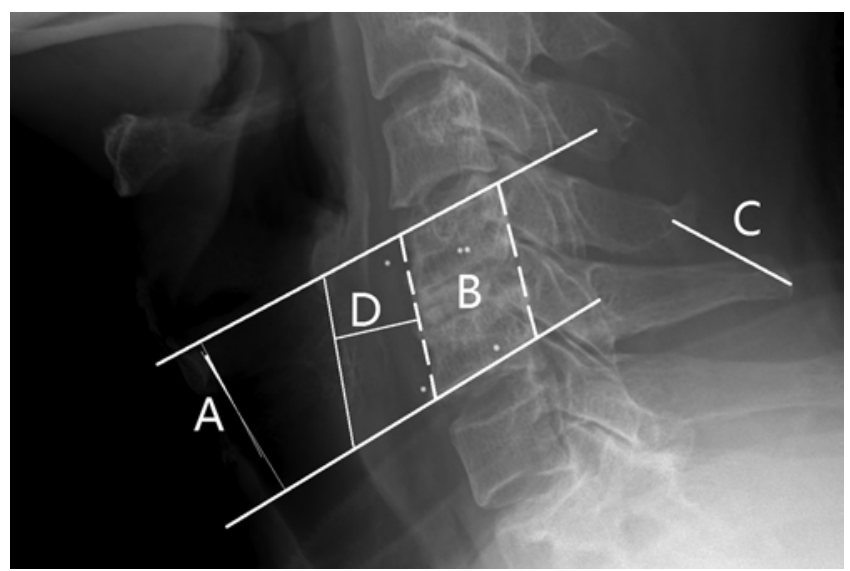

FIG. 2. Lateral radiograph measurements. Cobb's angle (A), intervertebral height (B), dynamic spinous process (C), and PVST (D) were evaluated. Intervertebral height was determined as an average of the anterior and posterior column measurements. The soft-tissue reaction was assessed by measuring the thickness of retropharyngeal tissue over time.

(SDs). Statistical evaluation was performed using SPSS version 18.1 (SPSS Institute Inc.). Paired sample t-tests and chi-square tests were used for data analysis, and we considered a $\mathrm{p}<0.05$ to be a significant difference.

\section{Results}

\section{Patient Demographics}

Over a 2-year period, we followed all 31 patients (38 segments) treated using a bACP and 47 patients (57 segments) treated using an mACP. All surgical procedures were performed between the levels of C3-4 and C6-7. Single-level procedures were performed in 61 patients and 2-level procedures in 17 patients. The mean follow-up time was $13.5 \pm 0.9$ months (range $12-18$ months) in the bACP group and 14.8 \pm 1.5 months (range 14-22 months) in the mACP group.

\section{Clinical Outcomes}

Clinical outcome based on the mJOA, VAS, and Odom's criteria was assessed for all of the patients. The mean mJOA score and mean VAS score for neck and arm pain at each time point are listed in Table 2. At 3 months postoperatively, the mean mJOA and VAS scores had significantly $(\mathrm{p}<0.05)$ improved to satisfactory levels in both groups. Moreover, at the latest follow-up, clinical symptom relief was observed as well. According to Odom's criteria, 24 patients $(77.4 \%)$ in the bACP group achieved an excellent outcome; 5 patients (16.1\%), a good

TABLE 2. Treatment outcomes according to VAS and mJOA scores*

\begin{tabular}{|c|c|c|c|c|c|c|}
\hline \multirow[b]{2}{*}{ Scoring System } & \multicolumn{2}{|c|}{ Preop } & \multicolumn{2}{|c|}{ 3-Mos Postop } & \multicolumn{2}{|c|}{ 1-Yr Postop } \\
\hline & bACP & mACP & $\mathrm{bACP}$ & mACP & bACP & $\mathrm{mACP}$ \\
\hline VAS & $6.3 \pm 1.2$ & $5.9 \pm 1.2$ & $1.5 \pm 0.7$ & $1.8 \pm 0.5$ & $1.3 \pm 0.2$ & $1.2 \pm 0.3$ \\
\hline mJOA & $10.7 \pm 1.2$ & $9.7 \pm 1.1$ & $14.8 \pm 1.2$ & $14.5 \pm 1.1$ & $15.2 \pm 1.1$ & $15.9 \pm 1.0$ \\
\hline
\end{tabular}

* Values are presented as means \pm SD. 
TABLE 3. Treatment outcomes according to Odom's criteria*

\begin{tabular}{lcccccc}
\hline & \multicolumn{2}{c}{3 -Mos Postop } & & \multicolumn{2}{c}{ 1-Yr Postop } \\
\cline { 2 - 3 } \cline { 5 - 6 } Outcome & bACP & mACP & & bACP & mACP \\
\hline Excellent & 19 & 27 & & 24 & 35 \\
\hline Good & 7 & 13 & & 5 & 9 \\
\hline Fair & 5 & 7 & & 2 & 3 \\
\hline Poor & 0 & 0 & & 0 & 0 \\
\hline
\end{tabular}

* Values are numbers of patients.

outcome; and 2 patients (6.5\%), a fair outcome. Comparatively, in the $\mathrm{mACP}$ group, the distribution was 35 patients $(74.5 \%)$ with an excellent outcome, 9 (19.1\%) with a good outcome, and $3(6.4 \%)$ with a fair outcome. No patients in either group experienced a poor outcome. Overall, $93.5 \%$ (29/31) of patients in the bACP group and 93.6\% (44/47) of patients in the mACP group had a favorable (excellent or good) outcome at the last follow-up before publication of this study, without statistically significant differences (Table 3).

\section{Radiographic Results}

\section{Bony Union and Intervertebral Height}

The fusion rate achieved after at least 6 months was $94.7 \%$ in the bACP group (36/38 segments in 31 patients), whereas this rate was $96.5 \%$ in the mACP group (55/57 segments in 47 patients; Fig. 3).

The intervertebral heights at the final follow-up, which corresponded to differences in the preoperative measurement values, were $1.47 \mathrm{~mm}$ for the bACP group and 2.02 $\mathrm{mm}$ for the $\mathrm{mACP}$ group. Although the intervertebral height remained significantly increased at the last followup, a decreasing trend was observed (Fig. 4).

\section{Sagittal Alignment}

The cervical alignment or spinal balance, as evaluated by measurement of Cobb's angle, improved significantly
( $\mathrm{p}<0.05)$ in both groups. However, a declining trend was noted during the observational period (Fig. 5).

\section{PVST Response}

The postoperative PVST response remained relatively constant over the study period (Figs. 6 and 7), with no significant changes between follow-up times.

\section{Complications}

No screw or plate breakage was observed during insertion, nor did any patients experience cerebrospinal fluid leakage or postoperative emergent tracheotomy.

An important related complication was persistent postoperative dysphagia accompanied by the occurrence of sterile abscesses. During our observations, only 2 patients endured such experiences; 1 patient's symptoms resolved 3 days after her operation. Another male patient complained of mild dysphagia even 4 weeks after the procedure, but his symptoms resolved by 3 months postoperatively without any intervention. The postoperative MRI also illustrated the absence of artifacts and prevertebral sterile abscesses (Fig. 7).

Other complications included wound pain and hoarseness, but these manifestations resolved during the patients' hospital stay.

\section{Discussion}

Since its introduction in the 1950s, ACDF has been one of the most commonly performed procedures to treat cervical spondylosis. An auxiliary titanium alloy plate can provide sufficient rigidity and stability to allow arthrodesis, prevent displacement or collapse of the intervertebral grafts, and maintain cervical lordosis to achieve a better prognosis. ${ }^{21}$ However, the excessive stiffness of the metallic plate may also shield some of the load necessary to achieve intervertebral bony union. Although semi-static and dynamic metal plates have been introduced, results have not been clearly established. ${ }^{5,25}$ Moreover, the sup-
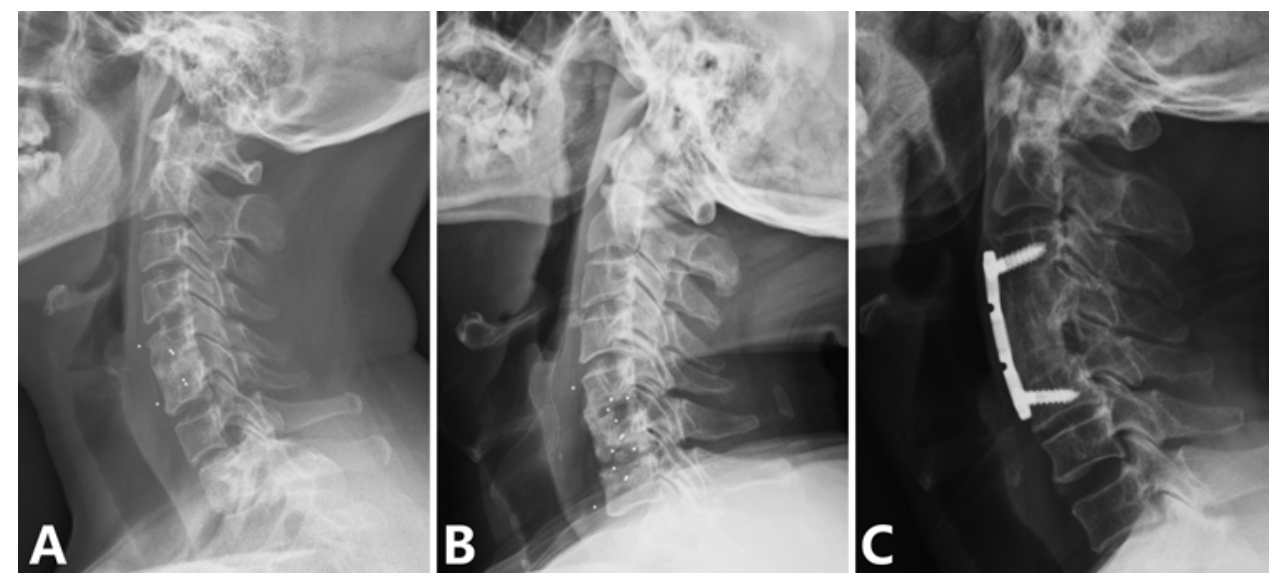

FIG. 3. A: Lateral radiograph obtained 15 months postoperatively in a 50 -year-old woman who had undergone a single-level ACDF using a bACP. B: Lateral radiograph obtained 16 months postoperatively in a 46-year-old man who had undergone a double-level ACDF using a bACP. C: Lateral radiograph obtained 18 months postoperatively in a 57-year-old woman who had undergone a double-level ACDF using an mACP. Evidence of continuous bony bridges across the graft-vertebral body interface was obvious, as was the absence of a radiolucent line in all the radiographs. 


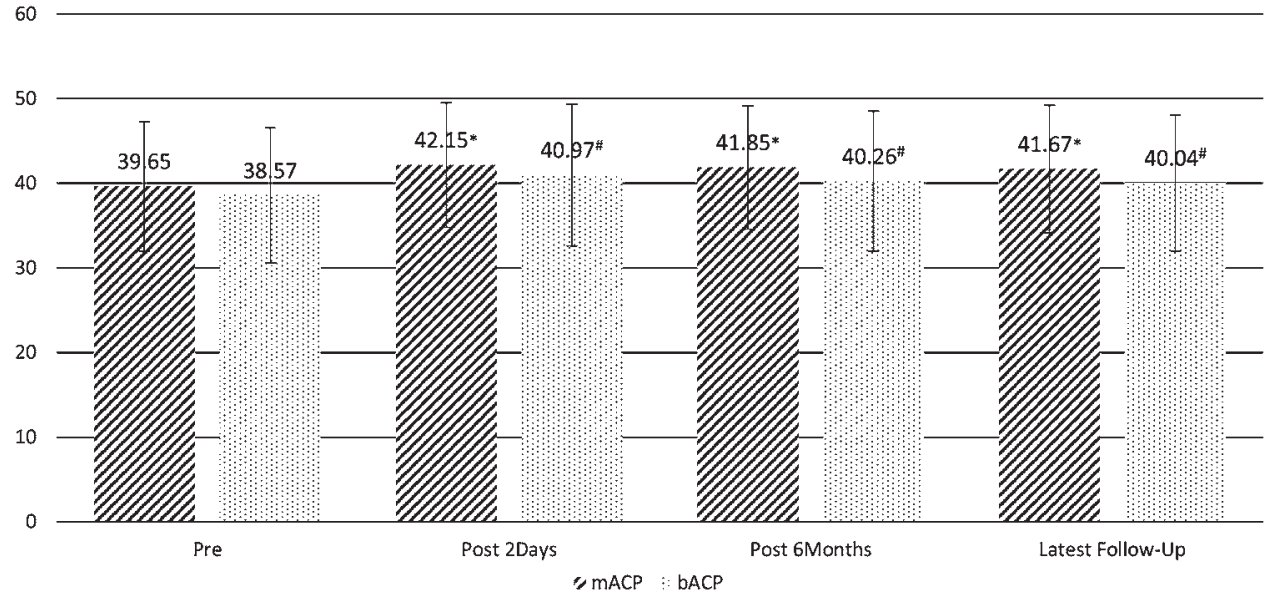

FIG. 4. Mean height of the intervertebral space over the course of the study. ${ }^{*} p<0.05$ compared with the preoperative value for the mACP group. $\# p<0.05$ compared with the preoperative value for the bACP group.

port offered by the implantation is theoretically needed only before bony union is achieved, and the permanence of the device may be harmful due to long-term complications such as breakage, migration, and soft tissue erosion. ${ }^{14,19}$ Additionally, the metallic alloy inevitably obscures postoperative MRI or CT results. ${ }^{2}$

To overcome these limitations, biodegradable plating systems were introduced for clinical application. In fact, before the use of an ACP in cervical spine surgery, biodegradable implants had established roles in craniomaxillofacial, neurological, general surgical, and orthopedic procedures. ${ }^{6}$ The poly-L-lactide-based implants degrade to lactic acid in vivo through a hydrolytic reaction, and complete absorption requires about $1.5-2$ years. ${ }^{1,6,10}$ However, studies have reported that at 6 months after implantation, the plates retain approximately $90 \%$ of their initial strength; ${ }^{1}$ thus, the durability of the plating system allows adequate opportunity for bony union. In addition, the relatively fragile and gradually weakening biodegradable plates (relative to titanium counterparts) transfer a greater burden to the grafting space and contribute to better arthrodesis. ${ }^{6}$
Several studies of biodegradable plating systems have reported conflicting results in bony fusion rates. ${ }^{1-4,8,9,13}$, $15,16,18,20,23,24$ Vaccaro et al. ${ }^{23}$ described the first series of patients treated with a biodegradable anterior cervical mesh in 2002. The fusion rate was $77 \%$ (7/9 patients) at an average follow-up of 206 days for single-level ACDF without severe complications or revisions. In 2004, Park et al. ${ }^{18}$ reported outcomes for 26 patients treated with the same implantation device. The fusion rate in their study was 96.2\% (25/26 patients), with 1 patient requiring additional surgery because of hardware failure. Moreover, in 2007, Aryan et al. ${ }^{2}$ showed that radiographic union was achieved at 6 months postoperatively in 51 of $52(98.1 \%)$ patients in their multicenter study. One asymptomatic patient had presented evidence of nonunion on flexion-extension imaging. Similarly, at 6 months postoperatively, radiographic fusion was seen in $92.3 \%$ of 30 patients in a 2009 study by Tomasino et al. ${ }^{20}$

In contrast, however, Bindal et al. ${ }^{3}$ reported a $12.5 \%$ (3/24) rate of pseudoarthrodesis with resorbable cervical plates, while the rate in the control titanium plate group was only $2.2 \%$ (2/93). Lebl et al. ${ }^{13}$ reported that radio-

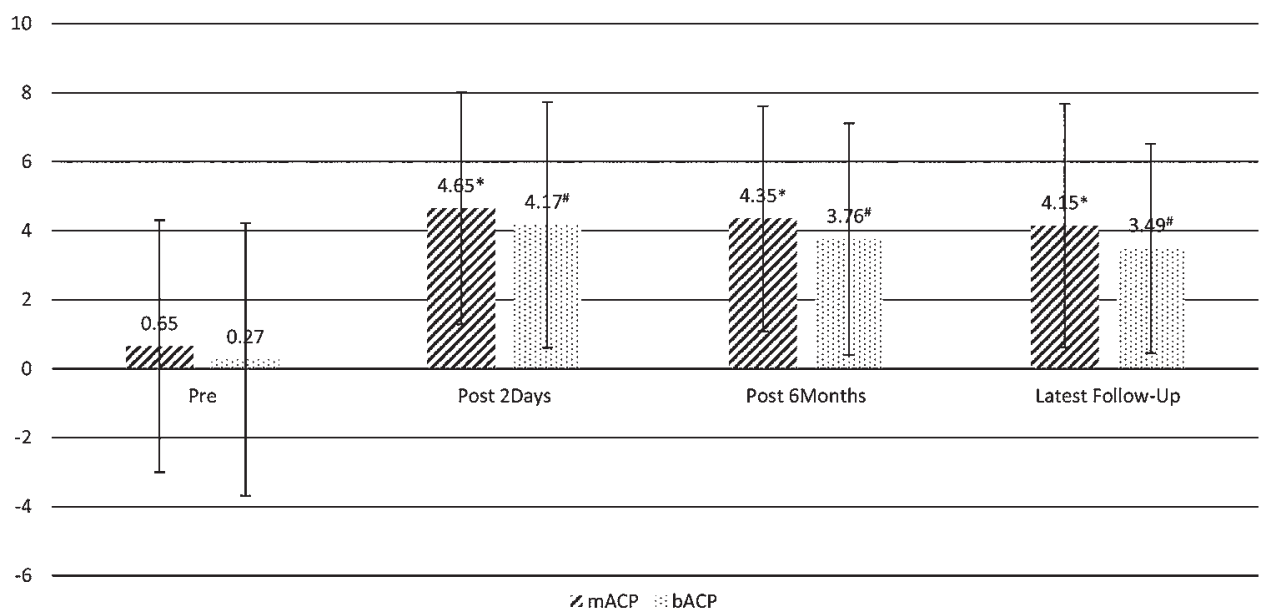

FIG. 5. Mean Cobb's angle as a measure of cervical sagittal alignment over the study period. ${ }^{*} p<0.05$ compared with the preoperative value for the mACP group. $\# p<0.05$ compared with preoperative value for the bACP group. 


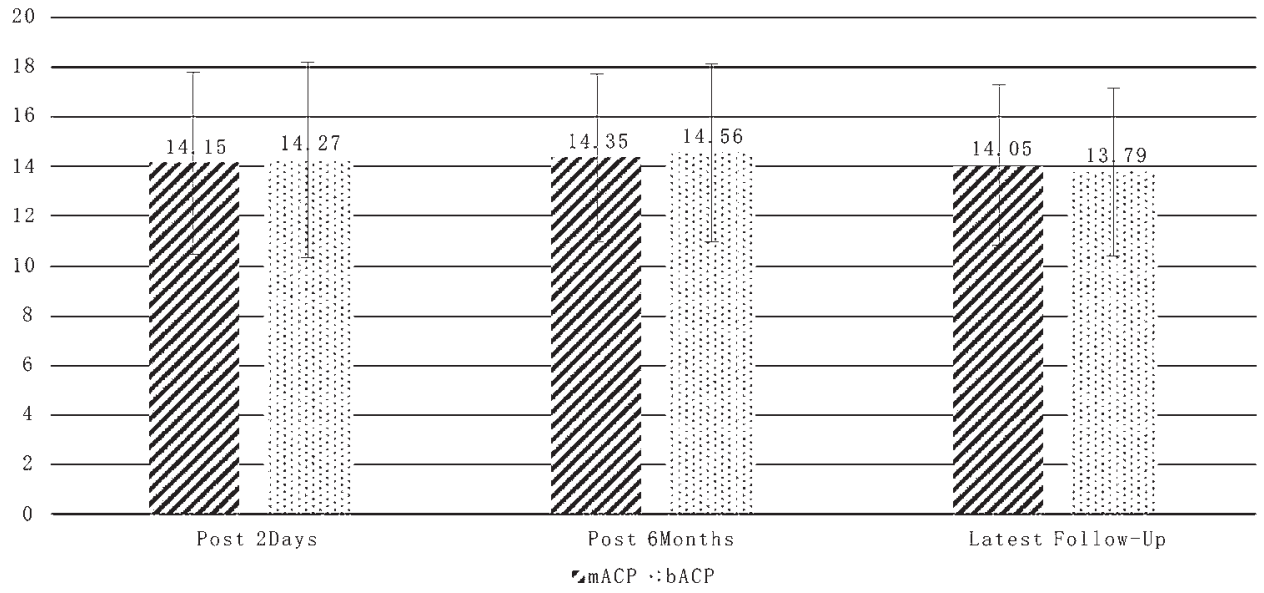

FIG. 6. Changes in PVST measured in radiographic assessments.

graphs obtained at later follow-ups demonstrated pseudoarthrodesis in 4 of 14 patients (29\%) treated using biodegradable plates and in 0 of 15 patients treated with a metal plate. Graft extrusion was found in 3 of the 4 (75\%) cases of pseudoarthrodesis.

We performed, to our knowledge, the first ACDF procedure using a bACP in China and herein present the first clinical and radiographic observations. The fusion rate was $94.7 \%$ (36/38 segments) with only 2 patients showing partial absence of bony bridging across the interbody space. However, the patients were asymptomatic and refused a secondary intervention. This result was considered very satisfactory and was comparable with results in the mACP group (96.5\%, 55/57 segments). Additionally, no evidence of graft extrusion was noted. Because there were no significant differences in the demographic and operational variables between the 2 groups, we can attribute the successful graft containment and bony union to the stability offered by the biodegradable plating system in both single- and double-level procedures.

A growing number of researchers have reported concerns regarding the reduced rigidity of the biodegradable material and doubt whether bACPs can provide sufficient strength for maintaining cervical intervertebral height and preserving physiological lordosis, which are of great concern for better long-term efficacy and prognosis. Although a multitude of preceding studies have provided encouraging results for the use of bACPs, $, 2,8,16,18,20,23$ other studies echo these concerns. Brkaric et al. ${ }^{4}$ reported early failure of the Mystique plate in 3 of 6 patients who underwent single-level ACDF. Bindal et al. ${ }^{3}$ attributed an increase in kyphotic angulation to inadequate rigidity or unrecognized fracture of the implanted bACP. In a more recent study in 2011, Lebl et al. ${ }^{13}$ showed that cervical lordosis was maintained in only 3 of 14 patients (21\%) in the bACP group compared with 8 of 15 patients (53\%) in the metallic plating group. Additionally, screw breakage during insertion was reported by Tomasino et al. ${ }^{20}$ and Brkaric et al. ${ }^{4}$ Of greater significance, these inferior radiographic manifestations were associated with worse clinical outcomes. ${ }^{13}$ In an ovine model using biodegradable plates ${ }_{15}^{15}$ although no radiographic evidence of device failure was noted, at necropsy, 6 of the 12 specimens had either a broken screw or a cracked plate, and these gross observations were further confirmed via histological staining.

However, in the present study, no breakage of plates or screws was identified intraoperatively. A decreasing trend was observed for intervertebral height and Cobb's angle measurements at the latest time point in the bACP group, compared with measurements in the mACP group, which

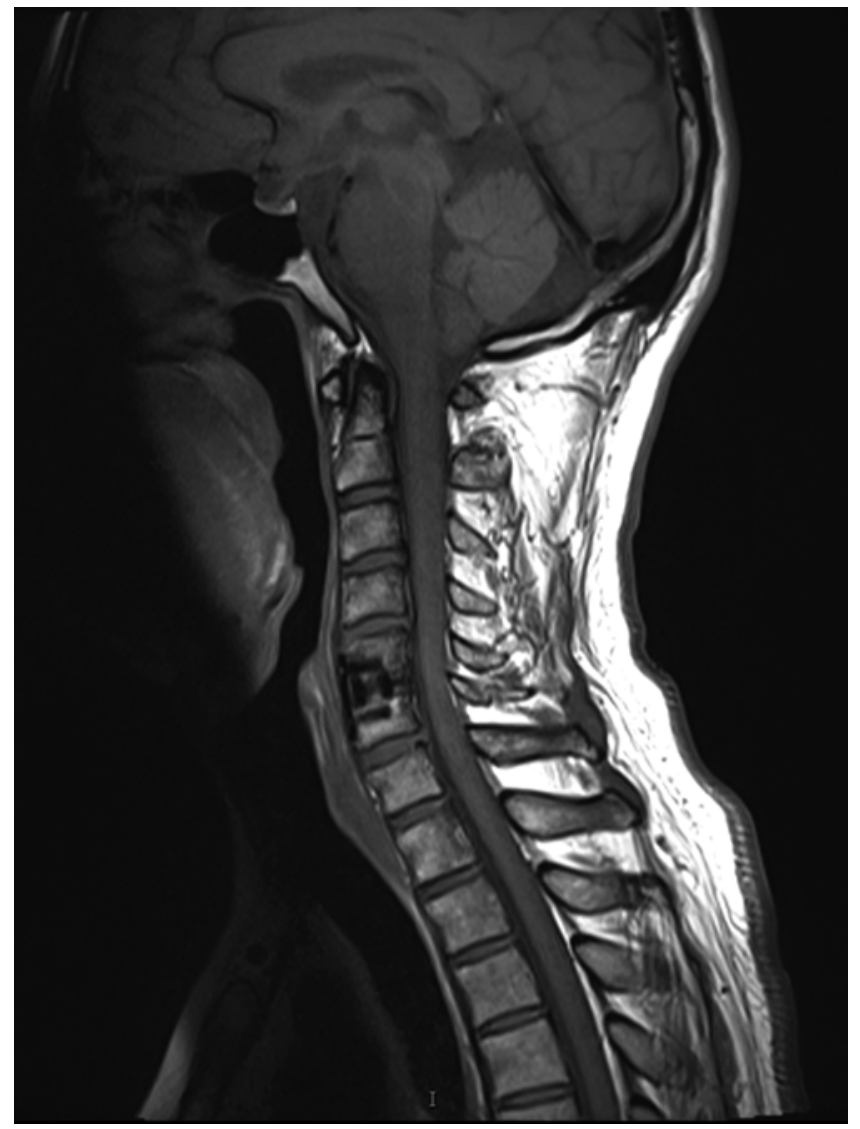

FIG. 7. MR image obtained 17 months postoperatively in a 47-year-old man who had undergone a single-level ACDF using a bACP, showing the absence of artifacts and prevertebral sterile abscesses. 
might be attributed to the fragility of the bACP; however, the differences were not statistically significant. Maintained intervertebral height and improved sagittal alignment were still exhibited at every follow-up postoperatively, which might indicate that absorbance of the bACP did not compromise the biomechanical stability required for bony union and for preserving physiological intervertebral height and Cobb's angle. More importantly, 93.5\% (29/31) of patients treated using a bACP obtained satisfactory clinical outcomes. Only 1 patient complained of the recurrence of preoperative symptoms, but these symptoms did not persuade him to undergo a second procedure.

Furthermore, we performed the first series of 2-level ACDF procedures using biodegradable plates and screws in China. The patients also presented good clinical outcomes during the immediate and early periods, and postoperative anterior/posterior and lateral flexion-extension radiographs taken at the last follow-up illustrated significant improvements in intervertebral height and segmental alignment. Indeed, these superior results might be attributed to the limited duration of our observation and the small number of patients involved. In addition, the effect of the auxiliary 4-week fixation with a neck collar could not be excluded. Nevertheless, we could also postulate that the results are related to the satisfactory stability introduced by the bACP. The favorable clinical outcomes made it impossible for us to further investigate invisible device breakage via histological or electron microscopy techniques; however, even if it existed, the outstanding bony union rate and promising recovery made it meaningless. Additionally, although hardware failure of absorbable plates appears to occur early after the procedure or intraoperatively, metallic devices can cause complications at any time after implantation. ${ }^{11,14,19,20}$ Lyons et al. ${ }^{15}$ stated that the sheep cervical spine is subject to different angular forces than the human spine because of obvious differences between quadrupeds and bipeds. Unlike sheep, humans often wear a cervical collar postoperatively to further immobilize the spine during healing and fusion.

Although numerous experimental and clinical studies have demonstrated the biocompatibility of the biodegradable material used in ACPs, ${ }^{2,8,16,20,24}$ the PVST reaction still deserves careful attention. In 2006, Vaccaro et al. ${ }^{22}$ demonstrated that MRI assessment of a bACP implantation at more than 2 years' follow-up did not show any inflammation. However, 4 patients were reported to have a symptomatic local aseptic reaction, and 3 of them required reoperation for evacuation of the collected fluid..$^{13,20}$ In our study, no secondary operation was needed for either emergent dyspnea or fluid evacuation. Transient dysphonia and dysphagia were noted in 7 of 31 patients in the bACP group, but the symptoms completely resolved uneventfully. Prophylactic wound drainage systems might be a contributing factor, but the absence of irritative symptoms 1 month postoperatively still confirmed the biocompatibility of the bACP system.

Additionally, the radiolucent biodegradable material does not produce any artifacts on MRI or CT scans, allowing for better interpretation (Fig. 7). The transparent nature of the biodegradable material provides reassurance about the precise location of the implant intraoperatively. ${ }^{2}$
Given that the biodegradable material will be completely hydrolyzed within 2 years, the use of a bACP renders removal of the plating system via a second or revision surgery unnecessary, thus improving the safety of the treatment strategy. ${ }^{2}$

There were several limitations in our study. It was a small and short retrospective observation, and long-term outcomes remain to be clearly determined. The difference in age between the 2 groups may generate considerable bias in the results pertaining to clinical and radiographic outcomes. In fact, 3 patients under 40 years of age were intentionally treated using a bACP system to prevent adjacent segment degeneration. Further studies of more patients need to be conducted to clarify this issue. Additionally, the influence of the more fragile biodegradable plates on preservation of the angulation and height of the intervertebral space requires assessment over a longer time period.

\section{Conclusions}

Although mild graft resorption was noted and the prospective efficacy of biodegradable instrumentation remained to be elucidated with longer-term observation, the overall encouraging early clinical and radiographic outcomes and acceptable complication rates in this study suggest that bACPs are alternative instruments in ACDF.

\section{References}

1. Ames CP, Cornwall GB, Crawford NR, Nottmeier E, Chamberlain RH, Sonntag VK: Feasibility of a resorbable anterior cervical graft containment plate. J Neurosurg 97 (4 Suppl):440-446, 2002

2. Aryan HE, Lu DC, Acosta FL Jr, Härtl R, McCormick PW, Ames CP: Bioabsorbable anterior cervical plating: initial multicenter clinical and radiographic experience. Spine (Phila Pa 1976) 32:1084-1088, 2007

3. Bindal RK, Ghosh S, Foldi B: Resorbable anterior cervical plates for single-level degenerative disc disease. Neurosurgery 61 (5 Suppl 2):305-310, 2007

4. Brkaric M, Baker KC, Israel R, Harding T, Montgomery DM, Herkowitz HN: Early failure of bioabsorbable anterior cervical fusion plates: case report and failure analysis. J Spinal Disord Tech 20:248-254, 2007

5. Campos RR, Botelho RV: Systematic review of the effect of dynamic fixation systems compared with rigid fixation in the anterior cervical spine. Eur Spine J 23:298-304, 2014

6. Ciccone WJ II, Motz C, Bentley C, Tasto JP: Bioabsorbable implants in orthopaedics: new developments and clinical applications. J Am Acad Orthop Surg 9:280-288, 2001

7. Emery SE, Bohlman HH, Bolesta MJ, Jones PK: Anterior cervical decompression and arthrodesis for the treatment of cervical spondylotic myelopathy. Two to seventeen-year follow-up. J Bone Joint Surg Am 80:941-951, 1998

8. Franco A, Nina P, Arpino L, Torelli G: Use of resorbable implants for symptomatic cervical spondylosis: experience on 16 consecutive patients. J Neurosurg Sci 51:169-175, 2007

9. Freeman AL, Derincek A, Beaubien BP, Buttermann GR, Lew WD, Wood KB: In vitro comparison of bioresorbable and titanium anterior cervical plates in the immediate postoperative condition. J Spinal Disord Tech 19:577-583, 2006

10. Frost A, Bagouri E, Brown M, Jasani V: Osteolysis following resorbable poly-L-lactide-co-D, L-lactide PLIF cage use: a review of cases. Eur Spine J 21:449-454, 2012

11. Gazzeri R, Tamorri M, Faiola A, Gazzeri G: Delayed migra- 
tion of a screw into the gastrointestinal tract after anterior cervical spine plating. Spine (Phila Pa 1976) 33:E268-E271, 2008

12. Kadaňka Z, Bednař́́k J, Novotný O, Urbánek I, Dušek L: Cervical spondylotic myelopathy: conservative versus surgical treatment after 10 years. Eur Spine J 20:1533-1538, 2011

13. Lebl DR, Bono CM, Metkar US, Grottkau BE, Wood KB: Bioabsorbable anterior cervical plate fixation for single-level degenerative disorders: early clinical and radiographic experience. Spine J 11:1002-1008, 2011

14. Lu X, Guo Q, Ni B: Esophagus perforation complicating anterior cervical spine surgery. Eur Spine J 21:172-177, 2012

15. Lyons AS, Sherman BP, Puttlitz CM, Patel VV, Abjornson C, Turner AS, et al: Failure of resorbable plates and screws in an ovine model of anterior cervical discectomy and fusion. Spine J 11:876-883, 2011

16. Nabhan A, Ishak B, Steimer O, Zimmer A, Pitzen T, Steudel WI, et al: Comparison of bioresorbable and titanium plates in cervical spinal fusion: early radiologic and clinical results. J Spinal Disord Tech 22:155-161, 2009

17. Odom GL, Finney W, Woodhall B: Cervical disk lesions. J Am Med Assoc 166:23-28, 1958

18. Park MS, Aryan HE, Ozgur BM, Jandial R, Taylor WR: Stabilization of anterior cervical spine with bioabsorbable polymer in one- and two-level fusions. Neurosurgery 54:631-635, 2004

19. Schmidt M, Maxime V, Pareire F, Carlier R, Lawrence C, Clair B, et al: A lethal case of meningitis due to Lactobacillus rhamnosus as a late complication of anterior cervical spine surgery. J Infect 62:309-310, 2011

20. Tomasino A, Gebhard H, Parikh K, Wess C, Härtl R: Bioabsorbable instrumentation for single-level cervical degenerative disc disease: a radiological and clinical outcome study. J Neurosurg Spine 11:529-537, 2009

21. Tribus CB, Corteen DP, Zdeblick TA: The efficacy of anterior cervical plating in the management of symptomatic pseudoarthrosis of the cervical spine. Spine (Phila Pa 1976) 24:860-864, 1999
22. Vaccaro AR, Sahni D, Pahl MA, Harrop JS, Sharan AD, Venger BH, et al: Long-term magnetic resonance imaging evaluation of bioresorbable anterior cervical plate resorption following fusion for degenerative and traumatic disk disruption. Spine (Phila Pa 1976) 31:2091-2094, 2006

23. Vaccaro AR, Venger BH, Kelleher PM, Singh K, Carrino JA, Albert T, et al: Use of a bioabsorbable anterior cervical plate in the treatment of cervical degenerative and traumatic disk disruption. Orthopedics 25 (10 Suppl):s1191-s1199, 2002

24. Whitmore RG, Tykocinski ES, Sanborn MR, Welch WC: Bioresorbable anterior cervical plate device for multi-level degenerative disc disease: case report with 8-year follow-up. J Clin Neurosci 18:1736-1738, 2011

25. Zaïri F, Aboukais R, Thines L, Allaoui M, Assaker R: Relevance of expandable titanium cage for the treatment of cervical spondylotic myelopathy. Eur Spine J 21:1545-1550, 2012

\section{Disclosures}

The authors report no conflict of interest concerning the materials or methods used in this study or the findings specified in this paper.

\section{Author Contributions}

Acquisition of data: Chen, W Yang. Analysis and interpretation of data: Xu, Wang. Drafting the article: Liu, Chen, C Yang. Critically revising the article: Liu, Chen, W Yang. Reviewed submitted version of manuscript: Liu. Approved the final version of the manuscript on behalf of all authors: Liu. Statistical analysis: Chen, S Yang, Xu. Administrative/technical/material support: C Yang, Ye, Wang. Study supervision: Chen, Feng.

\section{Correspondence}

Xianzhe Liu, Department of Orthopaedics, Union Hospital, Tongji Medical College, Huazhong University of Science and Technology, 1037 Luoyu Rd., Wuhan 430022, China. email: chenmc0603@163.com. 